\title{
In vitro and in vivo biocompatibility and osteogenesis of graphene-reinforced nanohydroxyapatite polyamide66 ternary biocomposite as orthopedic implant material
}

\author{
This article was published in the following Dove Press journal: \\ International Journal of Nanomedicine \\ 13 July 2016 \\ Number of times this article has been viewed
}

\section{Shiyang Zhang' \\ Qiming Yang' \\ Weikang Zhao' \\ Bo Qiao' \\ Hongwang Cui' \\ Jianjun Fan ${ }^{2}$ \\ Hong $\mathrm{Li}^{3}$ \\ Xiaolin $\mathrm{Tu}^{4}$ \\ Dianming Jiang'}

'Department of Orthopedics, The First Affiliated Hospital of Chongqing

Medical University, ${ }^{2}$ Molecular

Medicine and Cancer Research

Centre, Chongqing Medical University,

Chongqing, ${ }^{3}$ College of Physical

Science and Technology, Sichuan University, Chengdu, ${ }^{4}$ Institutes of Life Sciences, Chongqing Medical University, Chongqing, People's Republic of China
Correspondence: Dianming Jiang Department of Orthopedics, The First Affiliated Hospital of Chongqing Medical University, No I Youyi Road, Yuzhong District, Chongqing 4000I6, People's Republic of China

Tel +86 23 890। I 202

$\mathrm{Fax}+862389011217$

Email jdm57I026@vip.163.com
Abstract: Graphene and its derivatives have been receiving increasing attention regarding their application in bone tissue engineering because of their excellent characteristics, such as a vast specific surface area and excellent mechanical properties. In this study, graphene-reinforced nanohydroxyapatite/polyamide66 (nHA/PA66) bone screws were prepared. The results of scanning electron microscopy observation and $\mathrm{X}$-ray diffraction data showed that both graphene and nHA had good dispersion in the PA66 matrix. In addition, the tensile strength and elastic modulus of the composites were significantly improved by $49.14 \%$ and $21.2 \%$, respectively. The murine bone marrow mesenchymal stem cell line $\mathrm{C} 3 \mathrm{H} 10 \mathrm{~T} 1 / 2$ exhibited better adhesion and proliferation in graphene reinforced nHA/PA66 composite material compared to the nHA/PA66 composites. The cells developed more pseudopods, with greater cell density and a more distinguishable cytoskeletal structure. These results were confirmed by fluorescent staining and cell viability assays. After C3H10T1/2 cells were cultured in osteogenic differentiation medium for 7 and 14 days, the bone differentiation-related gene expression, alkaline phosphatase, and osteocalcin were significantly increased in the cells cocultured with graphene reinforced nHA/PA66. This result demonstrated the bone-inducing characteristics of this composite material, a finding that was further supported by alizarin red staining results. In addition, graphene reinforced nHA/PA66 bone screws were implanted in canine femoral condyles, and postoperative histology revealed no obvious damage to the liver, spleen, kidneys, brain, or other major organs. The bone tissue around the implant grew well and was directly connected to the implant. The soft tissues showed no obvious inflammatory reaction, which demonstrated the good biocompatibility of the screws. These observations indicate that graphene-reinforced nHA/PA66 composites have great potential for application in bone tissue engineering.

Keywords: graphene, orthopedic biomaterials, cell differentiation, stem cells, bone tissue engineering

\section{Introduction}

The development of new materials in bone tissue engineering has been progressing dramatically. New products in this field require substrate materials that promote cell attachment, proliferation, and differentiation. ${ }^{1,2}$ Nanohydroxyapatite (nHA), which constitutes natural bone together with type I collagen, is now considered an ideal candidate for fabricating orthopedic implants because of its similarity to inorganic components of human hard tissues and its excellent bioactivity and biocompatibility. ${ }^{3-5} \mathrm{nHA}$ ceramics are clinically used to coat the surface of orthopedic implants and for biomedical 
applications as bone-repairing materials. ${ }^{6,7}$ However, due to its brittleness and poor mechanical strength, as well as fatigue failure, the applications of nHA are limited. ${ }^{8}$

Polymers, such as high-density polyethylene, polyetheretherketone, and polyamides, have apparent advantages such as good mechanical properties and processability. ${ }^{9,10}$ As a result, nHA has been incorporated into polymers to fabricate organic/inorganic composites, which have improved mechanical properties compared to nHA alone, for bone tissue engineering applications. Polylactic acid (PLA) is widely used in bone tissue engineering because of its biodegradability and thermal plasticity. The combination of nHA and PLA possesses improved mechanical properties and osteoconductivity. The compressive strength and Young's modulus of nHA/PLA nanocomposites have reached values of $155 \mathrm{MPa}$ and 3.6 GPa, respectively. ${ }^{11,12}$ Polycaprolactone (PCL) is another bioresorbable polymer with possible applications in bone and cartilage. nHA/PCL, with a compressive modulus of $\sim 3 \mathrm{MPa}$, supported the proliferation of mesenchymal stem cells (MSCs) and guided their osteogenic differentiation. ${ }^{13}$

nHA/polyamide66 (nHA/PA66) composite materials have shown improved mechanical properties compared to those based on PLA, PCL, and chitosan composites, and they do not generate degradation products that cause immune reactions. Previous studies have demonstrated the good bioactivity, osteoconductivity, and biocompatibility of nHA/PA66 composites. ${ }^{14,15}$ In addition, the elastic modulus of nHA/PA66 composites (3-5 GPa) is similar to that of human cortical bone (3-25 GPa), eliminating the stress-shielding effect of metal implants (70-300 GPa). ${ }^{14,16,17}$ For many years, nHA/ P66A has been used for fabricating intervertebral fusion devices and bone-defect-filling materials used in clinical surgery. A previous study showed that four years after implantation, nHA/PA66 implants directly connected with bone tissue without the immunological rejection and trabecular bone resorption caused by the stress-shielding effect. ${ }^{18}$

The successful application of nHA/PA66 composites in the abovementioned area encouraged us to explore a broader range of their possible applications. Although the nHA/ PA66 composites have improved mechanical properties compared to PLA, our previous studies revealed that the mechanical strength of nHA/PA66 composites is still insufficient. To overcome this difficulty, many recent studies have used toughening materials, such as glass fibers and carbon fibers, to enhance the mechanical properties of nHA/PA66 composites. ${ }^{17,19,20}$

Graphene is another typical additive used for toughening of polymer materials. ${ }^{21}$ Graphene has a two-dimensional honeycomb crystal structure of tightly packed SP2 hybridized carbon atoms ${ }^{22}$ and is known to be the thinnest, lightest, and strongest material. ${ }^{23}$ The large specific surface area of graphene allows the Young's modulus and storage modulus of polymer/graphene nanocomposites to be considerably enhanced. ${ }^{24,25}$ A large number of studies have shown that graphene-reinforced composites can promote the adhesion, growth, and osteogenic differentiation of stem cells. ${ }^{26-28}$ Therefore, this study added graphene as a toughening agent to nHA/PA66 to improve the mechanical properties and bioactivity of the composite materials. The influence of graphene on the adhesion, proliferation, and differentiation of MSCs was also investigated. Currently, to the best of our knowledge, reports regarding the in vivo application of graphene-reinforced polymer composites for bone tissue engineering are rare..$^{29,30}$ We prepared a graphene $(\mathrm{G}) / \mathrm{nHA} /$ PA66 bone screw, implanted it into dogs, and studied its in vivo biocompatibility. The results revealed that graphene effectively enhanced the mechanical properties of nHA/ PA66 composites, and demonstrated remarkable bioactivity and biocompatibility. The G/nHA/PA66 nanocomposites prepared in the present study have potential orthopedic applications.

\section{Materials and methods Synthesis of G/nHA/PA66}

Graphene-reinforced nHA/PA66 was prepared by means of a solution blend method and an injection molding method. ${ }^{31,32}$ First, PA66 (Ultramid A3K, BASF, Ludwigshafen, Germany) and nHA (Meilan Chemical Technology Co Ltd, Shanghai, People's Republic of China) were added to formic acid, and the mixture underwent vigorous mechanical stirring. In this process, the PA66 dissolved and nHA dispersed. Graphene (purchased from Hengqiu Graphene Technology Co Ltd, Suzhou, People's Republic of China, purity: $>95 \%$, thickness: $\sim 1.0 \mathrm{~nm}$ ) was added to formic acid separately to form a different mixture, which underwent ultrasonic dispersal treatment for 30 minutes. The two solutions were then blended and underwent ultrasonication, as well as vigorous mechanical stirring for 30 minutes. Then, ethanol was added to precipitate the composite phase. The nanocomposite was filtered, washed with ethanol, and dried at $25^{\circ} \mathrm{C}$. The following composites were prepared: PA66 only; PA66 and nHA (30\% w/w); and PA66, nHA (30\% w/w), and graphene $(1 \% \mathrm{w} / \mathrm{w})$. The above composites were added into a double screw extruder (TSE-30A, Ruiya, Nanjing Haisi Extrusion Equipment Co. Ltd., Jiangsu, People's Republic of China) for further processing at $265^{\circ} \mathrm{C}$. During this process, the 
nanocomposites were more thoroughly mixed. Finally, dumbbell-shaped sample bars for mechanical tests, sheets for cell culture, and bone screws ( $3.5 \mathrm{~mm}$ in diameter) were prepared according to the ISO 527-2-2012 $2^{33}$ standard using an injection molding machine (160/120C, BOLE Shuangma Machinery Co. Ltd., Zhengzhou, Henan, People's Republic of China).

\section{Characterization of G/nHA/PA66}

The acquired nanocomposite was crushed after being submerged in liquid nitrogen and surface-coated with gold; its cross-section was then observed by scanning electron microscopy (Nova NanoSEM 400, FEI, Hillsboro, OR, USA) with an accelerating voltage of $5 \mathrm{kV}$. Scanning X-ray diffraction microscopy was performed using an X-ray diffractometer (D/Max-2550, Rigaku, Tokyo, Japan). The tensile strength, bending strength, and compressive strength were tested by a mechanical testing instrument (Reger 3050, Reger Instrument Co. Ltd., Shenzhen, People's Republic of China) with a testing speed of $5 \mathrm{~mm} / \mathrm{min}$, following the guideline of American Standards for Testing and Materials standards. ${ }^{34,35}$ Five samples were subjected to each test. The testing condition was room temperature and $60 \%$ relative humidity.

\section{Cell proliferation}

A mouse MSC line (C3H10T1/2, Cell Bank of Chinese Academy of Sciences, Shanghai, People's Republic of China) was cultured on ultraviolet-disinfected G/nHA/PA66 and nHA/ PA66 tablets $(10 \times 10 \times 2 \mathrm{~mm})$ at a density of $4 \times 10^{5}$ cells $/ \mathrm{mL}$ in Dulbecco's Modified Eagle's Medium, with $1 \mathrm{~g} / \mathrm{L}$ glucose, L-glutamine, and sodium pyruvate, (Cellgro, Corning, NY, USA) containing $10 \%$ fetal bovine serum and $1 \%$ penicillinstreptomycin under $5 \%$ carbon dioxide at $37^{\circ} \mathrm{C}$. The medium was changed every 2 days. The cell viability was detected by Cell Counting Kit-8 (Dojindo, Kumamoto, Japan) 1, 3, and 7 days after the culture according to the manufacturer's instructions. Cell Counting Kit- 8 was added to the medium at $10 \mu \mathrm{L} /$ well, and the samples were incubated at $37^{\circ} \mathrm{C}$ for 2 hours. The absorbance at $450 \mathrm{~nm}$ was read with a microplate reader. Each assay was repeated three times.

\section{Cell morphology}

Cell morphology was examined by fluorescence staining. After being cocultured with the composite for 4, 24, or 72 hours, the cell samples were fixed with 4\% polyformaldehyde (Boster, Wuhan, People's Republic of China) incubated for 1 hour with fluorescein isothiocyanate-phalloidin (ActinTracker Green Kit, Beyotime, Jiangsu, People's Republic of China) in the dark and then incubated for 5 minutes with 2-(4-amidinophenyl)-6-indolecarbamidine dihydrochloride (Sigma-Aldrich, St Louis, MO, USA). Finally, the cytoskeleton was observed under laser confocal microscopy (FV1000, Olympus, Tokyo, Japan).

\section{Alizarin red staining and quantitative detection}

C3H10T1/2 cells were inoculated onto G/nHA/PA66 discs $(10 \times 10 \times 2 \mathrm{~mm})$ at $4 \times 10^{5}$ cells $/ \mathrm{mL}$ and were cultured in osteogenic differentiation medium containing $10 \mathrm{mM}$ $\beta$-glycerophosphate (Sigma-Aldrich), $10^{-8} \mathrm{M}$ dexamethasone (Sigma-Aldrich), and $0.2 \mathrm{mM}$ ascorbic acid (SigmaAldrich). The media was changed every 2 days. After being cultured for 7 and 14 days, the cells were washed twice with phosphate-buffered saline, fixed in $4 \%$ paraformaldehyde for 15 minutes, and rinsed with deionized water three times. Next, $1 \%$ alizarin red was added at $1 \mathrm{~mL} /$ well. The samples were incubated at room temperature for 10 minutes, quickly washed with deionized water three times, and observed under a microscope (TE2000-U, Nikon, Tokyo, Japan). Finally, $500 \mu \mathrm{L}$ of $10 \%$ acetic acid solution was added to each well to extract color, and the absorbance at $450 \mathrm{~nm}$ was measured with a microplate reader (Multiskan GO, Thermo Scientific, Waltham, MA, USA).

\section{Quantitative real-time polymerase chain reaction assay}

After the cells were cultured in osteogenic medium for 7 and 14 days, RNA was extracted by a total RNA kit (Omega Bio-Tek, Norcross, GA, USA). Complementary DNA was synthesized using a PrimeScript RT reagent kit (TaKaRa, Shiga, Japan) $\left(37^{\circ} \mathrm{C}\right.$ for 15 minutes and $85^{\circ} \mathrm{C}$ for 5 seconds). A quantitative reverse transcription-polymerase chain reaction (qRT-PCR) assay was performed using SYBR premix ex taq (TaKaRa) with a CFX connect real-time PCR detection system (Bio-Rad, Hercules, CA, USA). Primers were designed and synthesized by Sangon Biotech Co Ltd (Shanghai, People's Republic of China) using the Primer Premier software (PREMIER Biosoft, Palo Alto, CA, USA). The primer sequences were as follows: alkaline phosphatase (ALP): F-5'-ACACTCAAGGGAGAGGTCCA-3', R-5'-GAATAAAGGCAGAGCCAGGA-3'; and osteocalcin (OC): F-5'-CCGCCTACAAACGCATCTAT-3', R-5' CAGGGCAGAGAGAGAGGACA-3'. Glyceraldehyde- 3-phosphate dehydrogenase was used as an internal control. The relative expression of the target gene was calculated using the $2^{-\Delta \Delta \mathrm{Ct}}$ according to the $\mathrm{C}_{\mathrm{t}}$ value measured in previous reports. ${ }^{36}$ 


\section{Surgical procedure}

Eighteen adult dogs were randomly divided into two groups (nine in each group). In the experimental group, bone screws were implanted in the femoral condyle according to the method reported previously. ${ }^{19}$ First, 3\% pentobarbital (Sigma-Aldrich) intraperitoneal injections for anesthesia $(1 \mathrm{~mL} / \mathrm{kg})$ were given to the dogs. The lower limbs were shaved and disinfected. The knee was flexed, and a $5 \mathrm{~cm}$ paramedian incision was placed. In order to fully expose the surgical site, the skin, superficial fascia and deep fascia was opened layer by layer. The knee was then extended to allow patellar dislocation to expose the femoral condyle. Two pieces of G/nHA/PA66 screws were implanted from the lateral side of the condyle. The incision was rinsed with iodophor and saline, and was closed in a layered fashion. Bilateral knees were operated. No restriction on activities was enforced after the surgery. Eating and other general activities of the animals were observed. The animals were sacrificed 7, 28, and 120 days after the operation, and specimens were collected. The same surgery, but without implantation of screws, was performed in the sham operation control group. Ethical approval was obtained from the Animal Care and Ethics Committee of Chongqing Medical University of China. The housing facility is in keeping with Chinese national standard Laboratory Animal-Requirements of Environment and Housing Facilities (GB 14925-2010). The care of laboratory animals and animal experimental operation conformed to Chongqing Management Approach of Laboratory Animal (Chongqing government order No 195).

\section{Bone formation around the implant}

At 7 and 28 days after the operation, samples from the surgical site were scanned with a vivaCT 40 scanner (Scano Medical, Brüttisellen, Switzerland) (pixel size $19 \mu \mathrm{m}$, scan voltage $70 \mathrm{kVp}$, scan current $114 \mathrm{~A}$, integral time $250 \mathrm{~ms}$ ). After the micro-CT scanning, the results of relevant bone volume/trabecular volume and three-dimensional reconstruction were not obtained directly. Instead, engineering documents containing the raw data were generated by the scanner. After importing the engineering documents into the $\mu \mathrm{CT}$ software (Scano Medical, Brüttisellen, Switzerland), the relevant bone volume/trabecular volume was calculated, and three-dimensional reconstruction of the bone was performed. According to the software's instruction, auto-contouring tools were used to compute the bone volume within $1 \mathrm{~mm}$ from the implanted bone screw. The relevant bone volume/trabecular volume was calculated, and three-dimensional reconstruction of the bone was performed to allow more direct observations of the implant interface and surrounding bone growth. In addition, bone tissue samples with screw fixation were fixed in $4 \%$ paraformaldehyde and dehydrated with an alcohol concentration gradient. The samples were embedded in organic glass, formed into hard tissue sections using a cutting and grinding system (E300CP/400 CS, EXAKT Technologies, Inc., Oklahoma City, OK, USA), and stained with Gieson Van's stains. The bone-implant contact (BIC) was measured under a microscope and calculated as $\mathrm{BIC}=($ contact length between the bone and implant/implant length) $\times 100 \%$.

\section{Biocompatibility test}

At 120 days after surgery, the kidney, liver, spleen, brain, and soft tissue around the implanted screws were fixed in $4 \%$ paraformaldehyde, dehydrated, and embedded in paraffin. The samples were cut into sections and stained with hematoxylin and eosin to determine whether there was significant organ damage or a local inflammatory response at the implant.

\section{Statistical analysis}

The statistical analysis was performed using GraphPad Prism 6 for Windows 8 (GraphPad Software, Inc., La Jolla, CA, USA). The data were expressed as the mean \pm standard deviation. A $t$-test was used for comparison between the groups, and $P<0.05$ was considered statistically significant. All in vitro experiments were done in triplicate unless specified.

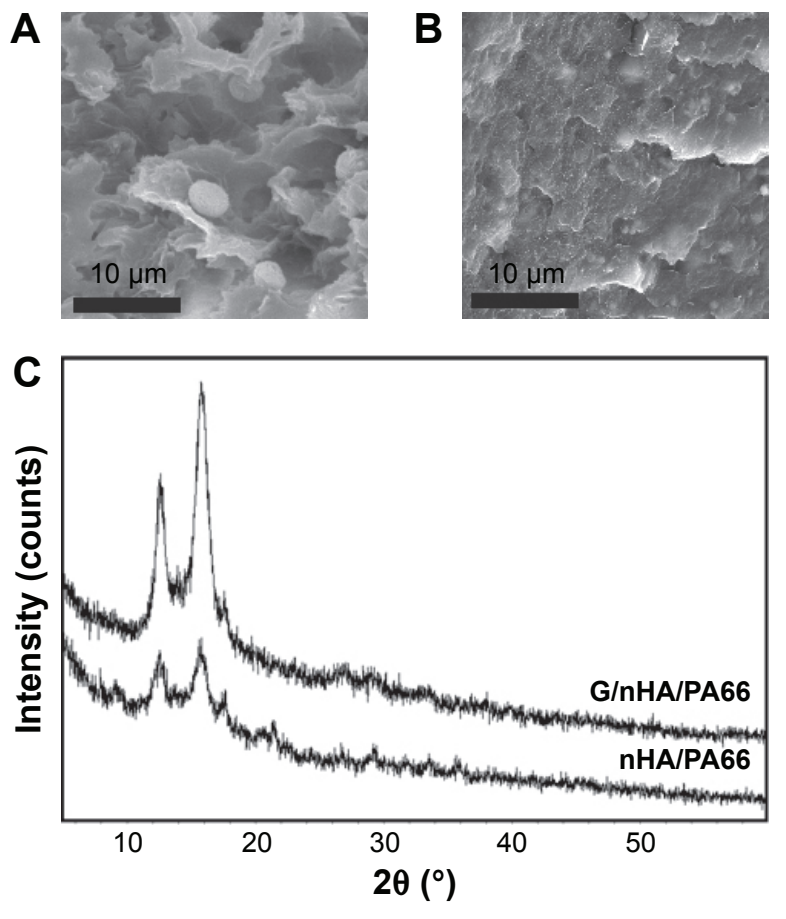

Figure I SEM micrographs of nHA/PA66 (A) and G/nHA/PA66 (B) and XRD patterns (C). (Magnification 2000×).

Abbreviations: G/nHA/PA66, graphene/nanohydroxyapatite/polyamide66; SEM, scanning electron microscope; $\mathrm{XRD}, \mathrm{X}$-ray diffraction;nHA/PA66, nanohydroxyapatite/ polyamide66. 
Table I Mechanical properties of G/nHA/PA66 composites

\begin{tabular}{llllll}
\hline Sample & $\begin{array}{l}\text { Bending strength } \\
(\mathbf{M P a})\end{array}$ & $\begin{array}{l}\text { Tensile strength } \\
\mathbf{( M P a )}\end{array}$ & $\begin{array}{l}\text { Breaking } \\
\text { elongation (\%) }\end{array}$ & $\begin{array}{l}\text { Elastic } \\
\text { modulus (GPa) }\end{array}$ & $\begin{array}{l}\text { Compressive strength } \\
\mathbf{( M P a )}\end{array}$ \\
\hline nHA/PA66 & $72.80 \pm 2.06$ & $41.61 \pm 0.40$ & $4.05 \pm 0.35$ & $2.76 \pm 0.28$ & $76.11 \pm 5.48$ \\
G/nHA/PA66 & $85.27 \pm 1.98^{*}$ & $62.06 \pm 0.52^{*}$ & $5.31 \pm 0.36^{*}$ & $3.60 \pm 0.24^{*}$ & $93.74 \pm 1.62^{*}$ \\
Human cortical bone 47,48 & $\sim 50-80$ & $\sim 50-150$ & $\sim 1-2$ & $\sim 3-20$ & $\sim 70-280$ \\
\hline
\end{tabular}

Note: $* p<0.05$ compared to the respective control ( $n=5$ specimens/group).

Abbreviations: G/nHA/PA66, graphene/nanohydroxyapatite/polyamide66; nHA/PA66, nanohydroxyapatite/polyamide66.

\section{Results}

\section{Characterization of materials}

Scanning electron microscope was used to observe the fracture surface morphology of the composites. A significant difference in nHA dispersion was observed between the composites with or without graphene. Agglomeration of HA (Figure 1A) was observed in nHA/PA66, whereas both graphene and HA were evenly dispersed in the PA66 matrix in G/nHA/PA66 (Figure 1B). The X-ray diffraction image of G/nHA/PA66 was almost identical to that of nHA/PA66. There was no characteristic graphene peak observed at 26 degrees (Figure 1C), suggesting good dispersion of graphene in the composite with no aggregation. ${ }^{37}$ The mechanical test results showed that the tensile strength and Young's modulus of G/nHA/PA66 were significantly increased compared to those of nHA/PA66 (Table 1).

\section{G/nHA/PA66 promotes cell adhesion and proliferation}

Cell viability assays were performed after the cells were cocultured with the composite materials for 1,3 , and 7 days. Figure 2 shows the optical density value of each group at different time points. The results show that the optical density value of each group increased over time. The group with graphene additives in nHA/PA66 exhibited higher metabolic activity than that of nHA/PA66 alone, indicating that the

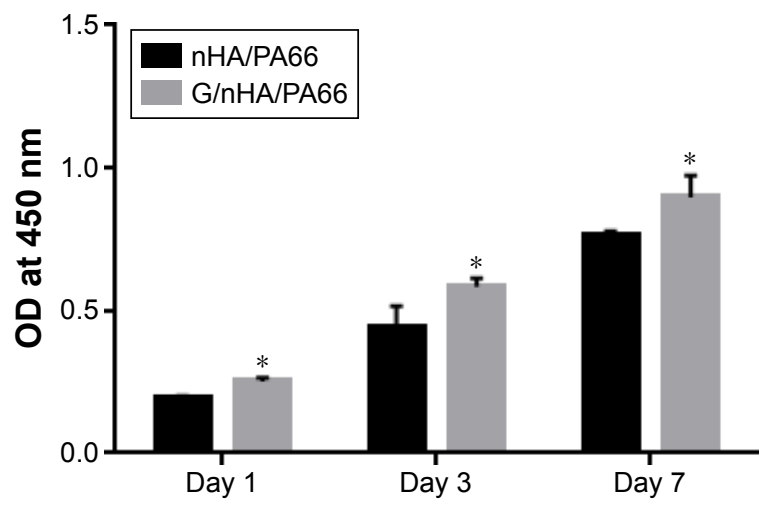

Figure 2 Cytotoxicity study of G/nHA/PA66 was performed by CCK-8 assay. Note: $* P<0.05$ compared to the respective control $(n=3)$.

Abbreviations: CCK-8, Cell Counting Kit-8; G/nHA/PA66, graphene/nanohydroxyapatite/polyamide66; nHA/PA66, nanohydroxyapatite/polyamide66; OD, optical density.
G/nHA/PA66 composite provided a better growth surface for $\mathrm{C} 3 \mathrm{H} 10 \mathrm{~T} 1 / 2$ cells.

Cell morphology was observed by immunofluorescence staining. After being cocultured with G/nHA/PA66 for 4 hours, the morphology of C3H10T1/2 was flat and the area large, indicating that the cell spreading is good. Many pseudopodium were observed in the cells, indicating that the cell extending is good, while the cells in the control group were smaller with fewer pseudopods. After coculturing for 24 and 72 hours, the cells showed increased density in both groups with good extending and spreading. Cells cocultured with G/nHA/PA66 exhibited a higher density and a significantly clearer cytoskeleton (Figure 3).

\section{G/nHA/PA66 promotes osteogenic differentiation}

Calcium salt deposition by $\mathrm{C} 3 \mathrm{H} 10 \mathrm{~T} 1 / 2$ cells during osteogenic differentiation was detected by an alizarin red staining assay. Cells were cocultured with G/nHA/PA66 and nHA/PA66 in osteogenic medium. After 7 and 14 days, a large area of orange staining was observed in the surrounding material, indicating the precipitation of calcium salts. No significant difference was observed between the two groups (Figure 4A-D). However, the quantitative detection results showed that the graphene-reinforced nHA/PA66 cocultured cells produced more calcium salt precipitation $(P<0.05)$ than the control group (nHA/PA66) (Figure 4E). This may be due to more calcium salts precipitating on the surface of the disc, which cannot be observed under a microscope; however, the color can be extracted and quantified.

At the molecular level, qRT-PCR was used to detect the osteogenic differentiation-related genes expression, ALP and OC. After the cells were cocultured with the composite materials for 7 or 14 days, the expression of ALP and OC was significantly upregulated $(P<0.05)$ in the G/nHA/PA66 cocultured cells compared to the control group (nHA/PA) (Figure 5).

\section{Good biocompatibility of G/nHA/PA66 implants in vivo}

At 120 days after G/nHA/PA66 implantation in vivo, histological observation of the liver, spleen, kidney, brain, and 


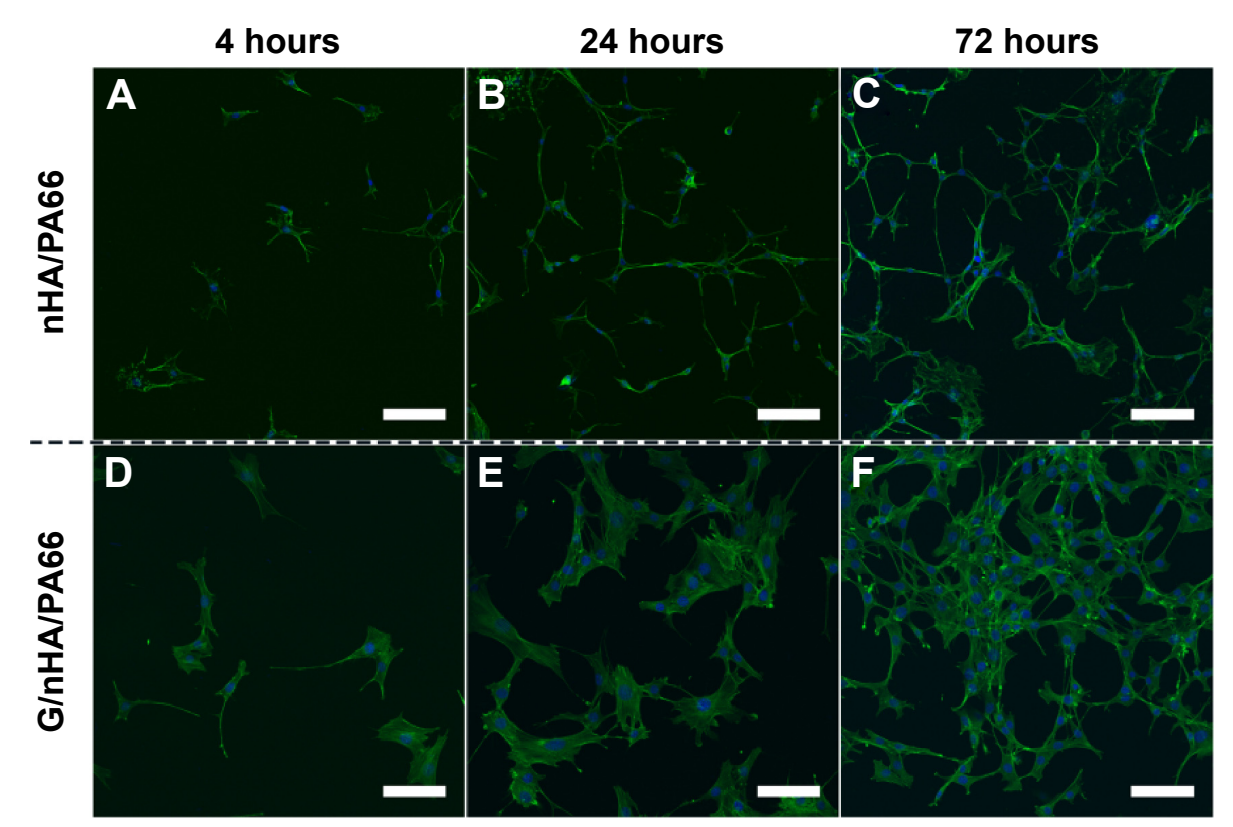

Figure 3 Confocal laser scanning microscope images of C3HI0TI/2 cells cultured on nHA/PA66 (A-C) and G/nHA/PA66 (D-F) discs (scale bar =l00 $\mu$ m, magnification 200x).

Abbreviations: G/nHA/PA66, graphene/nanohydroxyapatite/polyamide66; nHA/PA66, nanohydroxyapatite/polyamide66.

tissues around the implants was performed using hematoxylin and eosin staining. No significant inflammatory response was observed in the surrounding tissues. No significant abnormalities were found in the brains or other organs (Figure 6), although a previous study showed that graphene could pass across the blood-brain barrier. ${ }^{38}$

The process of bone growth around the implants was observed using histological staining and micro-computed tomography (micro-CT) scanning 7 and 28 days after the surgery. At 7 days after the surgery, a small amount of new bone formation was detected around the implant, and there was a gap of considerable size between the implant and the new bone. The BIC value was $26.80 \% \pm 7.84 \%$ (Figure 7). At 28 days after surgery, the trabecular bone was closely connected to the implant with fewer gaps, and the BIC value had reached $64.78 \% \pm 12.43 \%$ (Figure $7 \mathrm{C}-\mathrm{E}$ ). This observation was further confirmed by micro-CT scanning (Figure 8). At 28 days after surgery, the bone volume/ trabecular volume, trabecular number, and trabecular thickness had significantly $(P<0.05)$ increased by $122.6 \%$, $30.4 \%$, and $81.9 \%$, respectively, compared to 7 days after surgery (Figure 8).
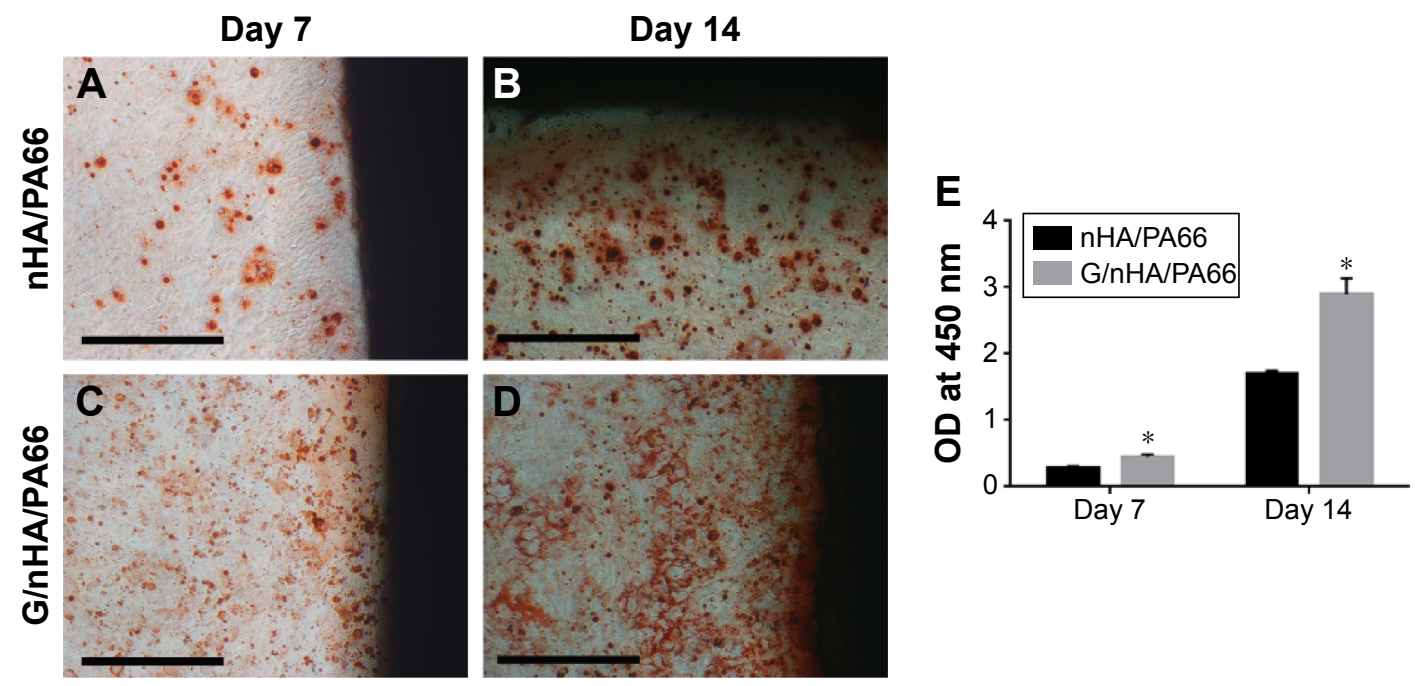

Figure 4 Alizarin red staining (A-D) and quantitative analysis (E).

Note: ${ }^{*} P<0.05$ compared to the respective control (scale bar $=200 \mu \mathrm{m}$, magnification $\left.200 \times\right)(n=5)$.

Abbreviations: G/nHA/PA66, graphene/nanohydroxyapatite/polyamide66; nHA/PA66, nanohydroxyapatite/polyamide66; OD, optical density. 


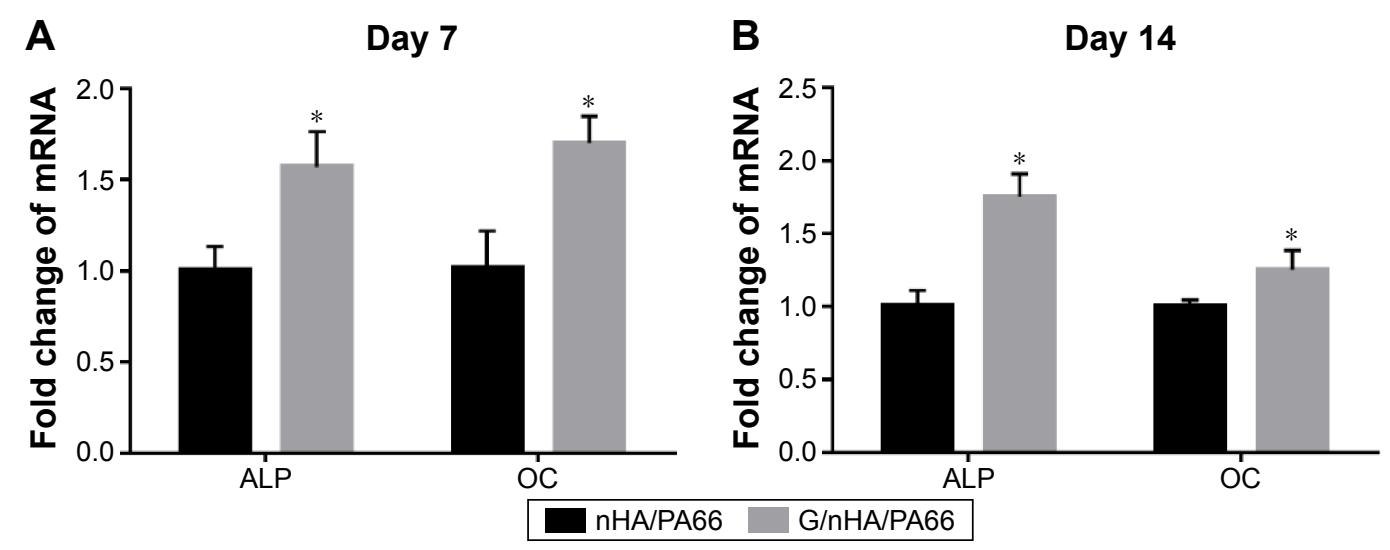

Figure 5 qRT-PCR analysis of the mRNA level of ALP and OC in C3HI0TI/2 cells cultured for 7 (A) and I4 (B) days.

Note: ${ }^{*} P<0.05$ compared to the respective control $(n=3)$.

Abbreviations: ALP, alkaline phosphatase; G/nHA/PA66, graphene/nanohydroxyapatite/polyamide66; mRNA, messenger RNA; nHA/PA66, nanohydroxyapatite/polyamide66; $O C$, osteocalcin; qRT-PCR, quantitative reverse transcription polymerase chain reaction.

\section{Discussion}

Bone has a complex layered structure that can be considered a type of composite material composed of type I collagen, hydroxyapatite, and water, in which inorganic and organic components account for $1 / 3$ and $2 / 3$ of the volume, respectively.,5 PA66 has a molecular structure similar to that of natural human collagen with good biocompatibility. ${ }^{39}$ Therefore, based on the above bionics theory, the application of nHA/PA66 composite was proposed. ${ }^{16}$ nHA/PA66 has good bioactivity and biocompatibility, and is nontoxic. nHA/PA66 has been used in clinical surgical implants, such as artificial bone and intervertebral fusion devices. . $^{15,40,41}$ However, due to its brittleness and poor mechanical properties, the application of nHA/PA66 composite was limited. To improve the mechanical properties of nHA/PA66 composite, carbon fiber and glass fiber have been added to nHA/PA66 as additives to enhance the mechanical strength of the composite materials..$^{17,19,20}$ In this study, graphene was used as an additive to enhance the mechanical properties of nHA/PA66 composite. The large specific surface area of graphene increased the interaction of the polymer materials, which enhanced the mechanical strength of the composite and increased the bioactivity of the material.

In recent years, graphene has received wide attention due to its electronic, mechanical, and thermal properties, and graphene is often used as an additive for toughening polymer composite materials. The folds on the outer surface of graphene can provide a space restriction at the nanometer scale, thereby enhancing its affinity for polymer materials. In addition, hydroxyl groups and other oxygen-containing functional groups in chemically modified graphene can form a chemical bond with polymer materials, thus greatly improving the load transfer between graphene and its substrates. ${ }^{37}$ Bao et $\mathrm{al}^{31}$ prepared G/PLA composite materials by adding graphene. Due to the comprehensive effects of graphene, such as the excellent mechanical properties, large specific surface area,
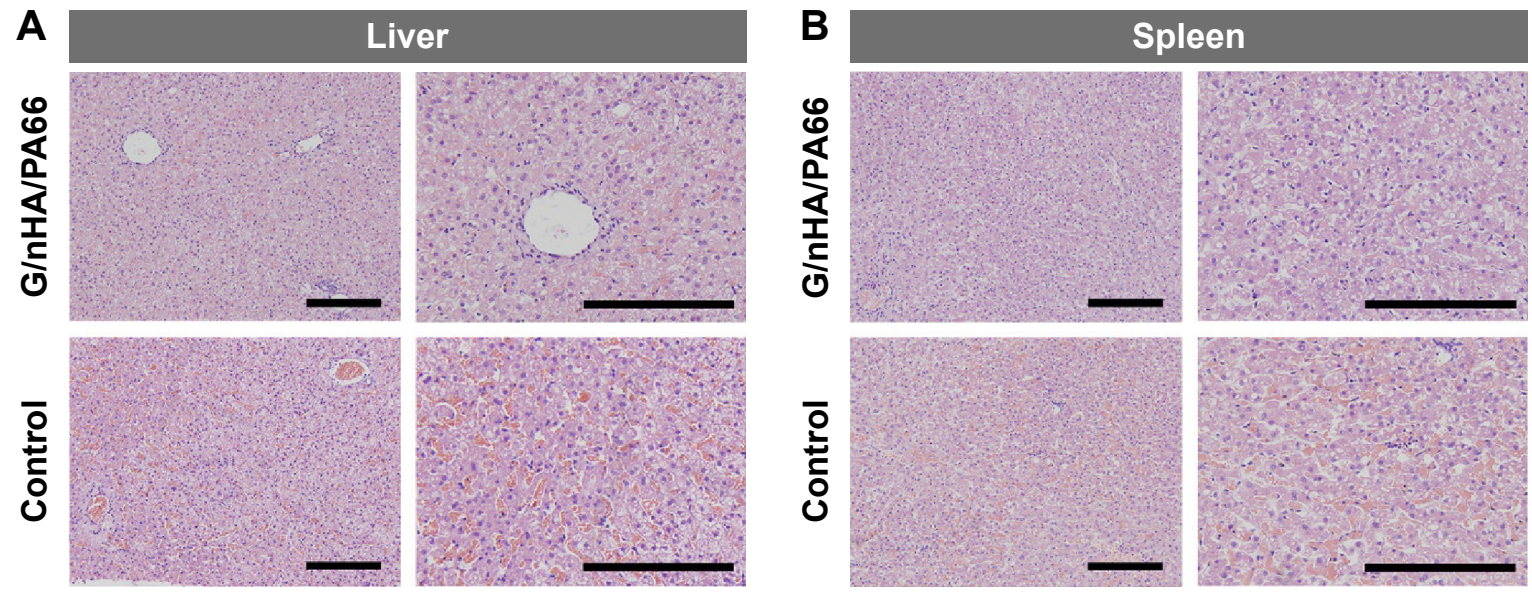

Figure 6 (Continued) 

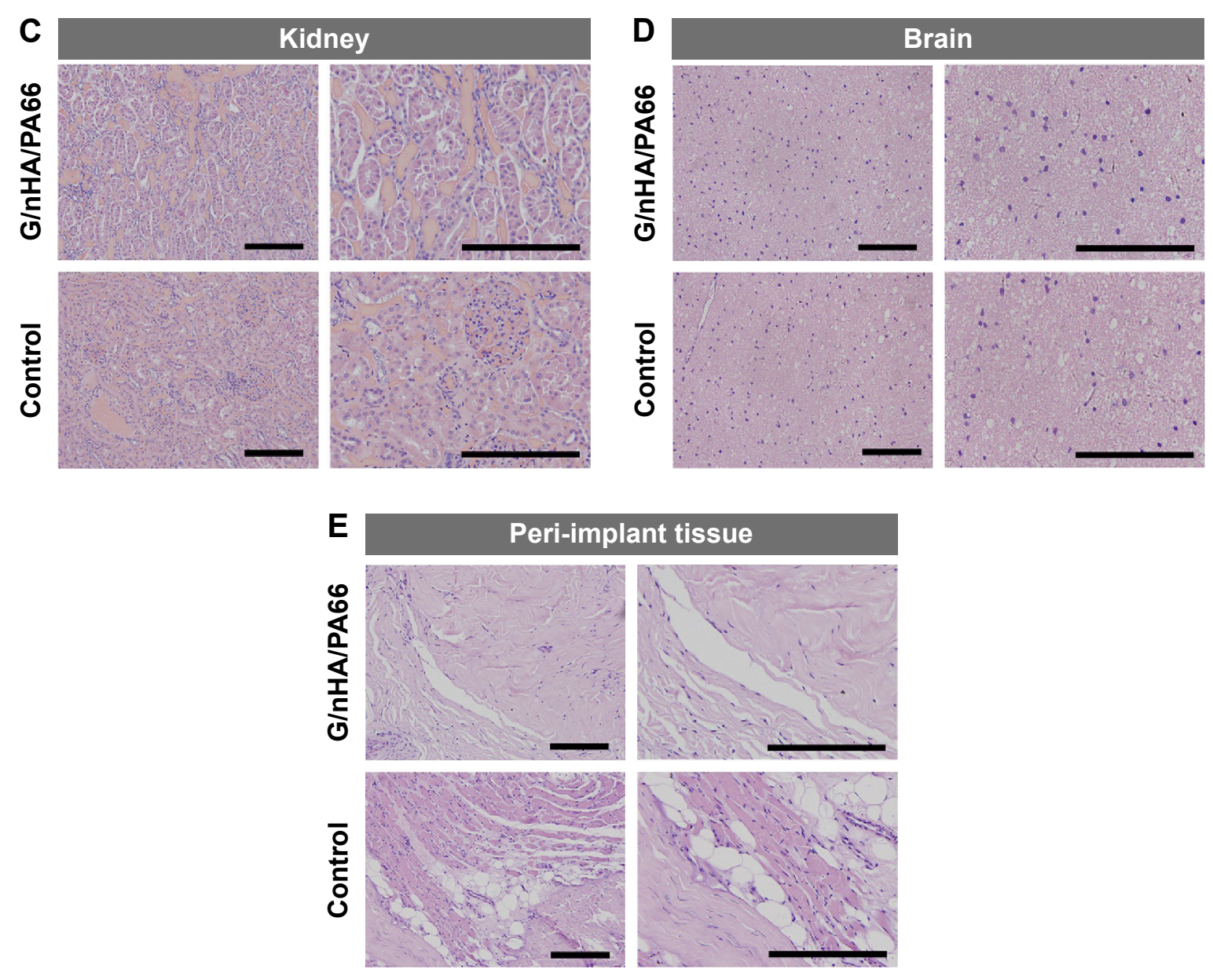

Figure 6 Gross histological analysis of liver (A), spleen (B), kidney (C), brain (D), and peri-implant tissue (E) I20 days after operation (scale bar $=200 \mu \mathrm{m}$, magnification I00 $\times$ ). Abbreviation: G/nHA/PA66, graphene/nanohydroxyapatite/polyamide66.
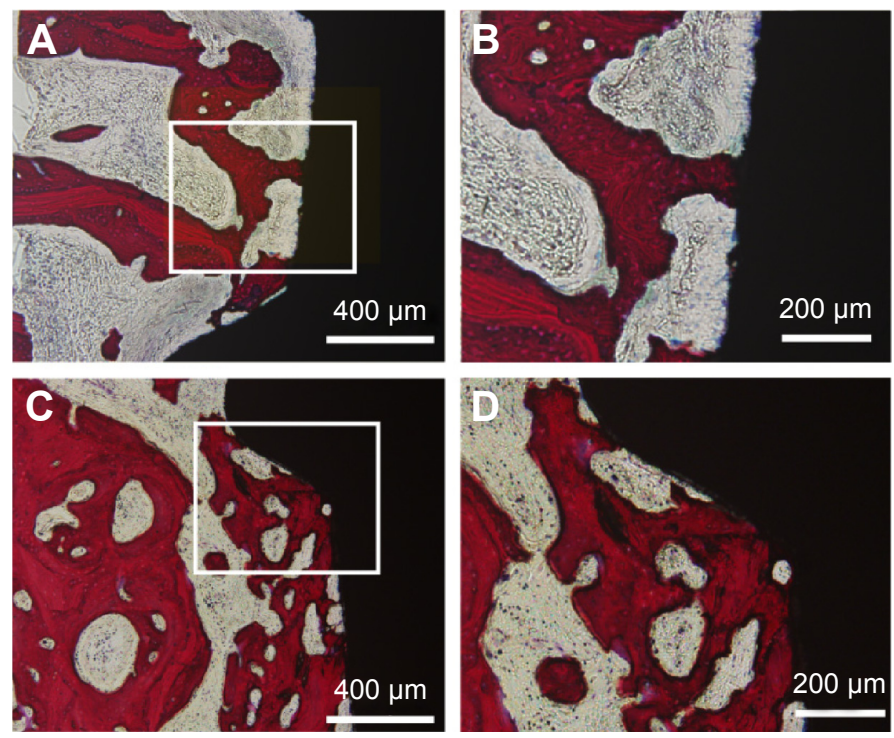

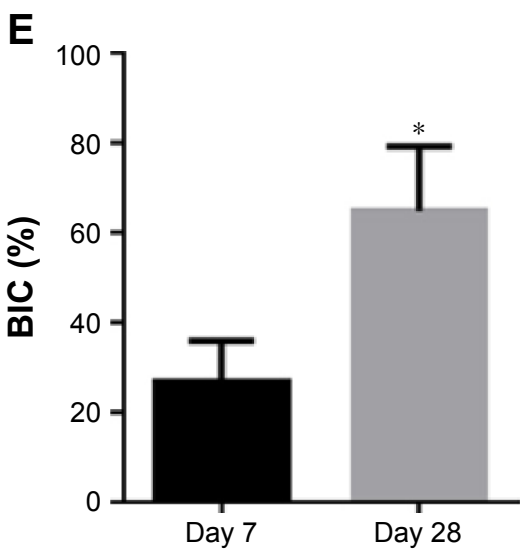

Figure 7 Histological observation of bone-implant interface for G/nHA/PA66 screws 7 (A, B) and 28 days (C, D) after operation (I00x). The BIC was calculated (E) Note: ${ }^{*} P<0.05$ compared to the respective control ( $n=4$ specimens/group).

Abbreviations: BIC, bone-implant contact; G/nHA/PA66, graphene/nanohydroxyapatite/polyamide66. 
A

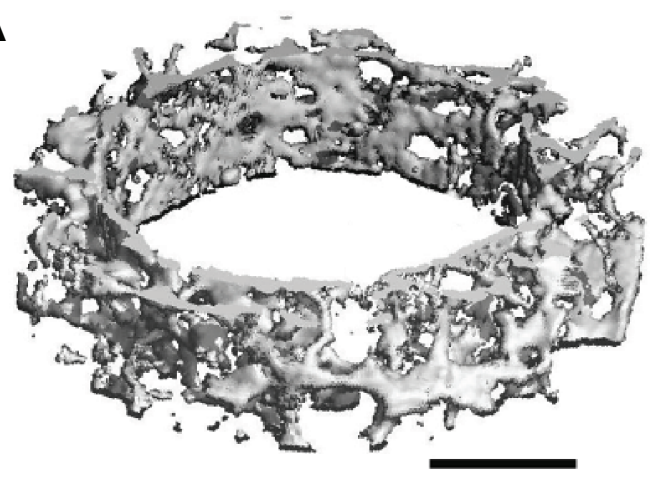

C
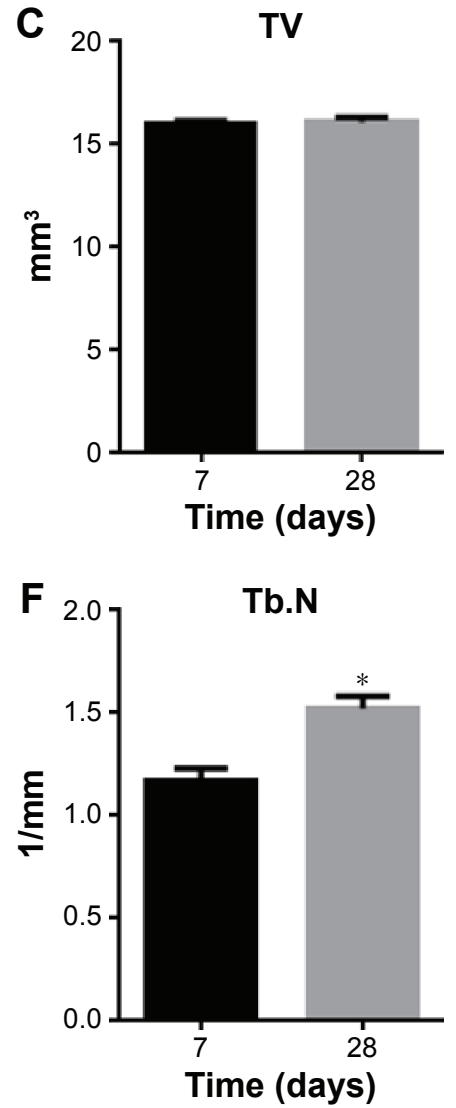

B

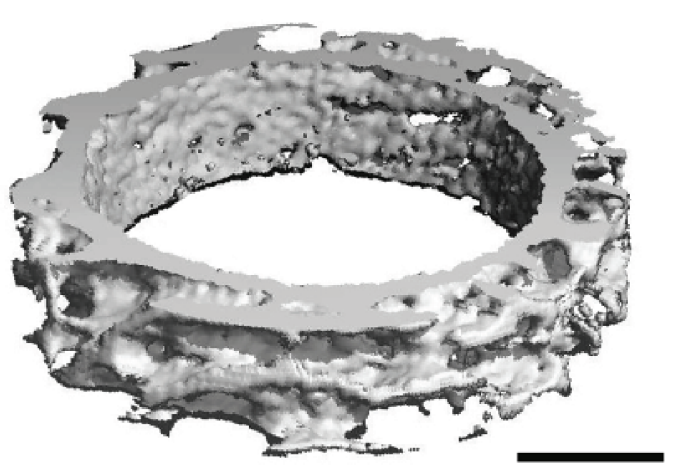

BV

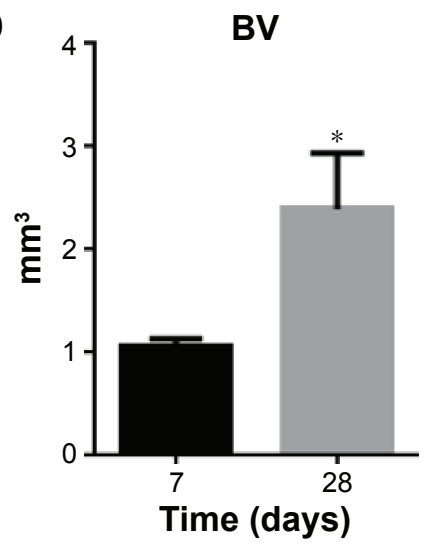

E

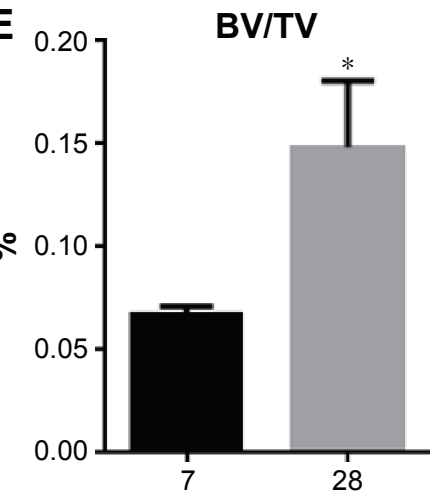

Time (days)
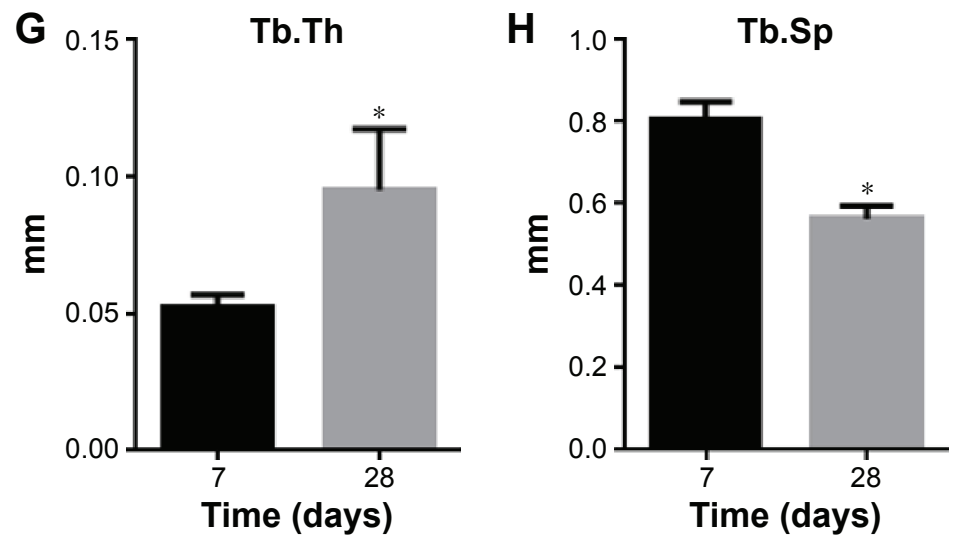

Figure 8 Micro-CT three-dimensional reconstruction of peri-implant bone with I mm with G/nHA/PA66 screws 7 (A) and 28 days (B) after operation. Peri-implant bone structural indices of $\mathrm{G} / \mathrm{nHA} / \mathrm{PA} 66$ materials $(\mathbf{C}-\mathbf{H})$.

Note: $* \mathrm{P}<0.05$ compared to the respective control ( $\mathrm{n}=5$ specimens/group).

Abbreviations: BV, bone volume; BV/TV, bone volume/trabecular volume; G/nHA/PA66, graphene/nanohydroxyapatite/polyamide66; Tb.N, trabecular number; Tb.Th, trabecular thickness; Tb.Sp, trabecular separation; TV, trabecular volume; micro-CT, micro-computed tomography.

and promotion of crystallization of the polymer, the material storage modulus was considerably improved. Marques et al ${ }^{32}$ studied the effects of different graphene contents on the mechanical properties of composites. The results showed that with the increase in the graphene content, the mechanical strength of the composite enhanced. However, when the content of graphene increased to a certain ratio, the mechanical strength weakened instead. A $1 \%$ mass fraction of graphene was suggested for maximum enhancement of the mechanical properties of these composites. The G/nHA/PA66 prepared in this study obtained a good dispersion for both graphene and nHA. Compared to nHA/PA66, the tensile strength increased by $49.14 \%$, and the bending strength by $17.11 \%$. Although the enhancement of this mechanical property was not exceptional and is still lower than metallic implant materials, ${ }^{33,42}$ the composite was close to meeting the material performance requirement for nonmetallic orthopedic implants. ${ }^{43}$

The application of graphene in the field of bone tissue engineering has drawn great attention not only because of its vast specific surface area, which can enhance the mechanical 
properties of polymer materials, but also because of its bioactivity, which can promote MSC adhesion, proliferation, and osteogenic differentiation. ${ }^{29}$ In this study, enhancing the nHA/PA66 composite with graphene led to enhanced cell spreading and extending. The cytoskeleton was more obvious, and the cell density was higher in early coculture stages. These results may be due to the unique nanotopography of the graphene-reinforced polymer composites increasing the expression of various integrins and connexins in MSCs. ${ }^{27,28}$ Another mechanism of graphene that can promote osteogenic differentiation is its provision of a concentrated platform for the chemical substances required for the induction of osteogenic differentiation. ${ }^{44}$ Strong $\pi-\pi$ stacking allows graphene to adsorb chemicals consisting of benzene rings, such as $\beta$-glycerophosphate and dexamethasone. Dexamethasone in combination with $\beta$-glycerophosphate plays an important role in upregulating the expression of osteogenic differentiation-related proteins and key enzyme genes. ${ }^{45,46}$ Additionally, insulin, which is an important chemical in the adipogenic differentiation process, is also adsorbed by graphene due to the $\pi-\pi$ stacking effect, which leads to insulin dysfunction after a conformational change upon graphene adsorption. This effect inhibited adipogenic differentiation while strengthening osteogenic differentiation in MSCs. ${ }^{44}$

Biocompatibility and noncytotoxicity are also very important properties for orthopedic implants. Numerous studies have thoroughly demonstrated the biocompatibility of nHA/PA66 composites in vivo and in vitro. ${ }^{9,16,19}$ However, the biological safety, especially in vivo safety, of graphenepolymer composites is controversial. There have been very few related studies on the in vivo safety of such composite materials; moreover, the results from these studies have not been consistent and are even contradictory. ${ }^{29}$ In this study, the liver, spleen, kidney, brain, and other major organs were investigated, and no obvious abnormalities were observed. The tissue surrounding the implant showed no inflammation. The bone around the implant tissue grew well. These results confirmed the good biocompatibility of G/nHA/PA66, which provides the basis for the feasibility of not removing the implant with a second operation. A long-term histocompatibility study of G/nHA/PA66 and studies of related molecular biological mechanisms still need to be conducted.

\section{Conclusion}

In this study, novel graphene-reinforced nHA/PA66 composites were prepared using a solution blending method and an injection molding method. The results demonstrated a uniform dispersion of graphene and nHA in the PA66 matrix. Compared to nHA/PA66 composites, the G/nHA/ PA66 composites showed significantly improved mechanical properties and bioactivity, which enhanced the adhesion, proliferation, and osteogenic differentiation of C3H10T1/2 cells; the composites also demonstrated good biocompatibility. In the in vivo implantation experiment, no major organ damage or inflammation around the implants was observed, which suggests a good histocompatibility of G/nHA/PA66. Therefore, G/nHA/PA66 exhibits great potential for orthopedic implant and bone tissue engineering applications.

\section{Acknowledgment}

This work was supported by National Natural Science Foundation of China (81501876) and Medical Scientific Research Foundation of Chongqing, China (2013-1-011).

\section{Disclosure}

The authors report no conflicts of interest in this work.

\section{References}

1. Bae H, Chu H, Edalat F, et al. Development of functional biomaterials with micro- and nanoscale technologies for tissue engineering and drug delivery applications. J Tissue Eng Regen Med. 2014;8(1):1-14.

2. Rose FR, Oreffo RO. Bone tissue engineering: hope vs hype. Biochem Biophys Res Commun. 2002;292(1):1-7.

3. Zhou H, Lee J. Nanoscale hydroxyapatite particles for bone tissue engineering. Acta Biomater. 2011;7(7):2769-2781.

4. Venkatesan J, Kim SK. Nano-hydroxyapatite composite biomaterials for bone tissue engineering - a review. J Biomed Nanotechnol. 2014;10(10): 3124-3140

5. Xu Q, Lu H, Zhang J, Lu G, Deng Z, Mo A. Tissue engineering scaffold material of porous nanohydroxyapatite/polyamide 66. Int J Nanomedicine. 2010;5:331-335.

6. Le Huec J, Schaeverbeke T, Clement D, Faber J, Le Rebeller A. Influence of porosity on the mechanical resistance of hydroxyapatite ceramics under compressive stress. Biomaterials. 1995;16(2):113-118.

7. McMahon RE, Wang L, Skoracki R, Mathur AB. Development of nanomaterials for bone repair and regeneration. J Biomed Mater Res B Appl Biomater. 2013;101(2):387-397.

8. Roeder RK, Converse GL, Kane RJ, Yue W. Hydroxyapatite-reinforced polymer biocomposites for synthetic bone substitutes. JOM. 2008; 60(3):38-45.

9. Huang D, Zuo Y, Li J, et al. Bioactive composite gradient coatings of nano-hydroxyapatite/polyamide66 fabricated on polyamide66 substrates. J R Soc Interface. 2012:rsif20110782.

10. Ma R, Tang S, Tan H, et al. Preparation, characterization, and in vitro osteoblast functions of a nano-hydroxyapatite/polyetheretherketone biocomposite as orthopedic implant material. Int J Nanomedicine. 2014;9: 3949-3961.

11. Yun Y-P, Kim S-J, Lim Y-M, et al. The effect of alendronate-loaded polycaprolactone nanofibrous scaffolds on osteogenic differentiation of adipose-derived stem cells in bone tissue regeneration. J Biomed Nanotechnol. 2014;10(6):1080-1090.

12. Zhang C, Lu H, Zhuang Z, Wang X, Fang Q. Nano-hydroxyapatite/ poly (L-lactic acid) composite synthesized by a modified in situ precipitation: preparation and properties. J Mater Sci. 2010;21(12): 3077-3083.

13. Heo SJ, Kim SE, Wei J, et al. Fabrication and characterization of novel nano- and micro-HA/PCL composite scaffolds using a modified rapid prototyping process. J Biomed Mater Res A. 2009;89(1):108-116. 
14. Jie W, Yubao L. Tissue engineering scaffold material of nano-apatite crystals and polyamide composite. Eur Poly J. 2004;40(3):509-515.

15. Xu H, Wang Y, Su X, Zhang X, Zhang X. Safety and efficiency of biomimetic nanohydroxyapatite/polyamide 66 composite in rabbits and primary use in anterior cervical discectomy and fusion. Int J Polym Sci. 2014;2014:696045.

16. Wang H, Li Y, Zuo Y, Li J, Ma S, Cheng L. Biocompatibility and osteogenesis of biomimetic nano-hydroxyapatite/polyamide composite scaffolds for bone tissue engineering. Biomaterials. 2007;28(22): 3338-3348.

17. Zhang $\mathrm{X}$, Zhang $\mathrm{Y}$, Zhang $\mathrm{X}$, et al. Mechanical properties and cytocompatibility of carbon fibre reinforced nano-hydroxyapatite/ polyamide66 ternary biocomposite. J Mech Behav Biomed Mater. 2015; 42:267-273.

18. Yang $\mathrm{X}$, Chen $\mathrm{Q}$, Liu L, et al. Comparison of anterior cervical fusion by titanium mesh cage versus nano-hydroxyapatite/polyamide cage following single-level corpectomy. Int Orthop. 2013;37(12):2421-2427.

19. Su B, Peng X, Jiang D, et al. In vitro and in vivo evaluations of nanohydroxyapatite/polyamide 66/glass fibre (n-HA/PA66/GF) as a novel bioactive bone screw. PLoS One. 2013;8(7):e68342.

20. Qiao B, Li J, Zhu Q, et al. Bone plate composed of a ternary nano-hydroxyapatite/polyamide 66/glass fiber composite: biomechanical properties and biocompatibility. Int J Nanomedicine. 2014;9: $1423-1432$.

21. Xu Z, Gao C. In situ polymerization approach to graphene-reinforced nylon-6 composites. Macromolecules. 2010;43(16):6716-6723.

22. Stankovich S, Dikin DA, Dommett GH, et al. Graphene-based composite materials. Nature. 2006;442(7100):282-286.

23. Lee C, Wei X, Kysar JW, Hone J. Measurement of the elastic properties and intrinsic strength of monolayer graphene. Science. 2008;321(5887): 385-388.

24. Zhao X, Zhang Q, Chen D, Lu P. Enhanced mechanical properties of graphene-based poly (vinyl alcohol) composites. Macromolecules. 2010;43(5):2357-2363.

25. Bao C, Guo Y, Song L, Hu Y. Poly (vinyl alcohol) nanocomposites based on graphene and graphite oxide: a comparative investigation of property and mechanism. J Mater Chem. 2011;21(36):13942-13950.

26. Chen GY, Pang DP, Hwang SM, Tuan HY, Hu YC. A graphene-based platform for induced pluripotent stem cells culture and differentiation. Biomaterials. 2012;33(2):418-427.

27. Kim J, Kim Y-R, Kim Y, et al. Graphene-incorporated chitosan substrata for adhesion and differentiation of human mesenchymal stem cells. J Mater Chem B. 2013;1(7):933-938.

28. Girase B, Shah JS, Misra RDK. Cellular mechanics of modulated osteoblasts functions in graphene oxide reinforced elastomers. Adv Eng Mater. 2012;14(4):B101-B111.

29. Gu M, Liu Y, Chen T, et al. Is graphene a promising nano-material for promoting surface modification of implants or scaffold materials in bone tissue engineering? Tissue Eng Part B Rev. 2014;20(5):477-491.

30. Dubey N, Bentini R, Islam I, Cao T, Neto AHC, Rosa V. Graphene: a versatile carbon-based material for bone tissue engineering. Stem Cells Int. 2015;2015:804213.

31. Bao C, Song L, Xing W, et al. Preparation of graphene by pressurized oxidation and multiplex reduction and its polymer nanocomposites by masterbatch-based melt blending. J Mater Chem. 2012;22(13): 6088-6096.
32. Marques PA, Gonçalves G, Singh MK, Grácio J. Graphene oxide and hydroxyapatite as fillers of polylactic acid nanocomposites: preparation and characterization. J Nanosci Nanotechnol. 2012;12(8): 6686-6692.

33. ISO 527-2. International Organization for Standardization; 2012. Available from: http://www.iso.org/iso/catalogue_detail. htm?csnumber=56046. Accessed February 2, 2012.

34. ASTM D695-96. American Society for Testing Materials; 1996. Available from: http://www.astm.org/DATABASE.CART/HISTORICAL/ D695-96.htm. Accessed April 12, 2013.

35. ASTM D638-14. American Society for Testing Materials; 2015. Available from: https://www.astm.org/Standards/D638.htm. Accessed February 20, 2015

36. Vimalraj S, Partridge NC, Selvamurugan N. A positive role of microRNA-15b on regulation of osteoblast differentiation. $J$ Cell Physiol. 2014;229(9):1236-1244.

37. Ramanathan T, Abdala A, Stankovich S, et al. Functionalized graphene sheets for polymer nanocomposites. Nat Nanotechnol. 2008;3(6): $327-331$.

38. Mendonça MC, Soares ES, de Jesus MB, et al. Reduced graphene oxide induces transient blood-brain barrier opening: an in vivo study. J Nanobiotechnology. 2015;13(1):78.

39. Pant HR, Risal P, Park CH, Tijing LD, Jeong YJ, Kim CS. Synthesis, characterization, and mineralization of polyamide-6/calcium lactate composite nanofibers for bone tissue engineering. Colloids Surf B Biointerfaces. 2013;102:152-157.

40. Xiong Y, Ren C, Zhang B, et al. Analyzing the behavior of a porous nano-hydroxyapatite/polyamide 66 (n-HA/PA66) composite for healing of bone defects. Int J Nanomedicine. 2014;9:485.

41. Zhao Z, Jiang D, Ou Y, Tang K, Luo X, Quan Z. A hollow cylindrical nano-hydroxyapatite/polyamide composite strut for cervical reconstruction after cervical corpectomy. J Clin Neurosci. 2012;19(4):536-540.

42. ISO 5832-1. International Organization for Standardization; 2007. Available from: http://www.iso.org/iso/home/store/catalogue_tc/ catalogue_detail.htm?csnumber=39023. Acccessed June 15, 2007.

43. ASTM F2026-07. American Society for Testing and Materials; 2007. Available from: http://www.astm.org/DATABASE.CART/ HISTORICAL/F2026-07.htm. Accessed January 17, 2013.

44. Lee WC, Lim CHY, Shi H, et al. Origin of enhanced stem cell growth and differentiation on graphene and graphene oxide. ACS Nano. 2011;5(9): 7334-7341.

45. Haynesworth S, Goshima J, Goldberg V, Caplan A. Characterization of cells with osteogenic potential from human marrow. Bone. 1992;13(1): $81-88$.

46. Oshina H, Sotome S, Yoshii T, et al. Effects of continuous dexamethasone treatment on differentiation capabilities of bone marrow-derived mesenchymal cells. Bone. 2007;41(4):575-583.

47. Reilly DT, Burstein AH. The mechanical properties of cortical bone. J Bone Joint Surg Am. 1974;56(5):1001-1022.

48. Vaz MF, Canhão H, Fonseca JE. Bone: a Composite Natural Material In: Těšinova $\mathrm{P}$, editor. Advances in Composite Materials - Analysis of Natural and Man-Made Materials. Rijeka: INTECH Open Access Publisher; 2011:195-228.
International Journal of Nanomedicine

\section{Publish your work in this journal}

The International Journal of Nanomedicine is an international, peerreviewed journal focusing on the application of nanotechnology in diagnostics, therapeutics, and drug delivery systems throughou the biomedical field. This journal is indexed on PubMed Central, MedLine, CAS, SciSearch ${ }^{\circledR}$, Current Contents ${ }^{\circledR} /$ Clinical Medicine,

\section{Dovepress}

Journal Citation Reports/Science Edition, EMBase, Scopus and the Elsevier Bibliographic databases. The manuscript management system is completely online and includes a very quick and fair peer-review system, which is all easy to use. Visit http://www.dovepress.com/ testimonials.php to read real quotes from published authors. 